\title{
Digital Simulation of Sewer Networks and Structures Operation in ANSYS CFX to Increase Energy Efficiency
}

\author{
Sviatoslav Fedorov \\ Department of Water use and Ecology, \\ Faculty of Environmental Engineering \\ and Municipal Services \\ Saint Petersburg State University of \\ Architecture and Civil Engineering \\ Saint Petersburg, Russia \\ svyatoslavfedorov@mail.ru
}

\author{
Alina Feskova \\ Department of Water use and Ecology, \\ Faculty of Environmental Engineering \\ and Municipal Services \\ Saint Petersburg State University of \\ Architecture and Civil Engineering \\ Saint Petersburg, Russia \\ alinafeskova@gmail.com
}

\author{
Veronika Asaul \\ Department of Construction Economics \\ and Housing and Utility Infrastructure \\ Saint Petersburg State University of \\ Architecture and Civil Engineering \\ Saint Petersburg, Russia \\ asaul@inbox.ru
}

\begin{abstract}
- the sewage networks functioning is accompanied by the processes of intensive emission of sewage gases (hydrogen sulfide, methane, ammonia, carbon dioxide) from the sewage stream into the air. Subsequent gas distribution due to the entraining stream ability results in gas accumulation in the under-roof space of pipelines and wells and subsequent seepage into the environment. This process reduces the functioning reliability and the environmental safety of the network and therefore requires preliminary forecasting during structures design and operation.

The purpose of the study: to create an Ansys CFX based model of the sewage network. Results: creation of the sewage network model taking into account two-phase flow of wastewater stream and a mixture of air and sewage gas (hydrogen sulfide), model calculation data - liquid and gas phases ratio in the design area, the pressure field in the gas-air environment, the content of hydrogen sulfide volume fraction in the design area. Practical significance: determination of places of intensive emission of hydrogen sulfide and accumulation places to justify measures for gas cleaning and to protect the walls of pipes and structures against gas corrosion.
\end{abstract}

Keywords-sewerage, digital modeling, Ansys CFX, hydrogen sulfide, gas emission rate, environmental safety, gas corrosion.

\section{INTRODUCTION}

City sewage collection and transportation systems generate and distribute toxic and foul-smelling sewage gases. The rate of sewage gas production and transportation in the under-roof space depends on the air-gas mixture flow rate in the system's pipelines. Currently, in the domestic engineering practice, sewage systems are designed only from the point of view of transporting the required wastewater flow rate without distribution of air-gas mixture streams. Therefore, to a greater extent, air motion is an uncontrollable and unpredictable process.

Currently, the process for designing any engineering structures is interfaced with the simulation of technological processes, since it allows observing the work of the future structure in all details. This approach allows to detect hidden defects and imperfections and to optimize the engineering structures as much as possible in terms of energy efficiency, reliability and safety of operating personnel. [1]
To solve two-phase problems, the following packages are currently most often used: Open Foam, Flow 3D, Flow Vision, which allows to most vividly see the mechanism of joint movement of liquid and gas, as well as to additionally solve heat and mass transfer problems.

The engineers do not use such packages so often in designing; however, the availability of skills to use such software will allow solving complex unique problems in real life setting.

The aim of this paper is to develop a new approach to design based on the system-theoretical methods that will be used by engineers designing and operating systems, as well as environmental engineers involved in research on odor and air pollution control near sewage facilities. The process calculation should take into account the hydraulic characteristics of the wastewater flow (non-silting rate, pipeline filling) [2], low-pressure entrainment of gas-air masses in the under-roof space of the pipeline, as well as the possible presence of natural or forced draft. This approach should be applicable both to newly designed systems and to real-life urban networks. The results of model calculations will certainly ensure reliable operation of sewage networks, increase environmental safety and moreover its energy efficiency.

The most interesting papers in this sphere are the results of Carrera L. [3], Wang Y. C. [4], Edwini-Bonsu S. [5], Lowe S. [6]. The paper of Carrera L. reflects the study of the gas emission mechanism in real conditions of the sewage network and the development of models for gas emission forecast. Other authors have studied the process of gas-air medium movement due to the enthralling stream ability and natural draft, as well as the network ventilation calculation methods are being developed. In Russia, in the field of network ventilation research, the papers of Vasiliev V.M. [7] and Malkov A.V. are best known. [8].

\section{STUDY METHODOLOGY}

\section{A. Construction of the calculation area of the sewage network section}

To obtain reliable results on calculating the gas-air mixture rate and directions of movement, it is necessary to have a pressure distribution mode in all network sections and 
structures. It is important to note that the complex aerodynamic calculation of pipelines and structures is quite complicated and today is performed on the basis of empirical dependencies obtained from observations at existing objects. The difficulties related to the calculation of network ventilation are primarily associated with the inconsistency of the boundary conditions which can greatly affect the system as a whole [8].

To simplify the approach, it is necessary to calculate a pressure in the under-roof space on all network sections, depending on the filling similarly to works $[9,10]$, which is a determining factor when moving the gas-air masses. Data on the vacuum gauge pressure in the pipeline section can be taken using the calculating charts described in the paper of Fyodorov S.V. and Telyatnikova A.M. CFX [11]. Similar pressure curves shall also be obtained for the sewage network structures (overflow wells, tanks of sewage pumping stations, etc.). Having obtained the network pressure distribution diagram, it will be possible to calculate the mass flow of air masses and to estimate sewage gas distribution and concentration.

For the purpose of subsequent approval of the method and regularities of the two-phase flow movement, it is proposed to carry out model calculations in ANSYS CFX for sewage networks of a significantly shorter length, but providing wastewater disposal similar to real structures, taking into account the effluents flow from outlets; movement through network sections; drop in overflow wells and discharge into the sewage pumping station tank followed by pumping out. Certainly, the results of this calculation will make it possible to most clearly follow up the gas-air medium movement mechanics and to evaluate the pressure changes along a sewage network length at the first approximation. The results of monitoring the existing network indicate the presence of vacuum pressure in the under-roof space at the network section [12,13,14], which decreases in wells with connections from outlets that provide a new portion of the air into the network.

For the study, a sewage network was simulated, which consists of two headers: main 1-6 and supply 8-6. In well No. 6 , runoff from two collectors is combined and sent to the sewage pumping station (well No. 7). Both headers are provided with wells that receive runoff from wastewater outlets (wells Nos. 1, 3, 8, 9, 10) and wells with a differential (No. 4, 11). All network sections have a diameter $\mathrm{dn}=200$ $\mathrm{mm}$. The outlets are accepted as $\mathrm{dn}=150 \mathrm{~mm}$. The length of all sections is $5 \mathrm{~m}$ (Fig. 1).

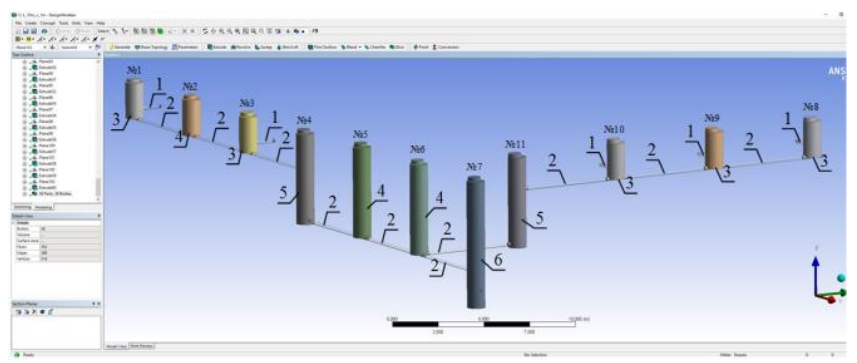

Fig. 1. Calculation area of the sewage network section 1 - outlet; 2 - pipeline section; 3 - inspection well with outlet (differential flow $0.5 \mathrm{~m}$ ); 4 - a viewing well; 5 - well with the pipe drop structure (drop flow $3 \mathrm{~m}) ; 6$ - sewage pumping station.
Fig. 1 shows the design of the internal network airspace, the pipe drop structure walls are made in the form of cutouts (voids). Pipe sections are separate ("frozen") objects. All elements (wells and pipe sections) are connected as a single part. For the area of wells, a tetrahedral pattern was built. For the pipe sections, the Sweep method was chosen to divide the space with hexahedron cells.

\section{B. Assignment of initial and boundary conditions}

The modeling process proposes water flow entering into all the outlets of the calculated area. Due to the flow entraining ability, the air also enters the calculation area. Any sewage gas can enter the water stream through the pipeline tray. The results of the study with incoming gas - hydrogen sulfide are presented as an example. The paper considered the solution of a dynamic problem until the steady motion is achieved along the movement of the water and gas-air medium. The gas starts flowing into the water stream from the moment of steady-state movement.

To simulate the inflow, the following initial and boundary conditions were established (Fig. 2). Two conditions were specified in the input section of the outlets. The first condition took into account the water flow and was determined using the Inlet parameter, which set the incoming flow rate $\mathrm{V}, \mathrm{m} / \mathrm{s}$, and filling $\mathrm{h} / \mathrm{d}$. The second condition took into account the by flow with setting the Opening parameter, in which the excess pressure was set equal to Pexc $=0 \mathrm{~Pa}$ (atmospheric pressure presence). It is important to note that for the first network version, the air flow through all outlets was simulated, and according to the second network version, the air flow was taken into account only through the first outlet. At the hatches border of the first downstream wells and the last one (SPS), the atmospheric pressure presence was also established. The hydrogen sulfide flow was set along the tray surface. For this purpose, at the stage of calculation area geometry creation, an additional edge was created in the form of a strip along the pipeline section of the pipeline tray. For this edge, the condition for entering mass flow rate Mgas, $\mathrm{kg} / \mathrm{s}$, was determined. The hydrogen sulfide flow rate in all sections is taken the same $0.01 \mathrm{~kg} / \mathrm{s}$ for the first network version and $0.005 \mathrm{~kg} / \mathrm{s}$ for the second version. The flow outlet with an admixture of hydrogen sulfide and an air mixture is determined by the Outlet condition with an excess pressure equal to Pexc $=0 \mathrm{~Pa}$. This condition is established for the branch from the SPS well.

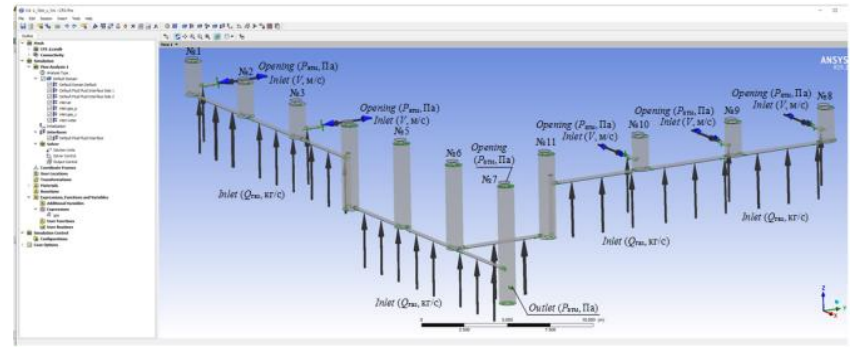

Fig. 2. Pattern of the boundary conditions of the sewer network model.

The outlet branch is adopted with a smaller diameter $\mathrm{dn}=$ $150 \mathrm{~mm}$ in order to reduce the pipeline throughput and to ensure fluid accumulation in the SPS tank. The condition of the presence of only air in the calculation area with excess pressure Pexc $=0 \mathrm{~Pa}$ is established as the initial state of the system (at time moment $\mathrm{t}=0 \mathrm{~s}$ ). The sections located 
normally to the flow and installed in each section of the header were assigned to record the simulation results in the calculation area. Excess pressure; mass flow rate of water and gas mixture are determined in the cross sections.

Qualitative verification of the calculation results was carried out according to the hydraulic calculation tables [14]. Upon reaching the steady-state movement, the water flow rate corresponding to the established slope and filling was recorded in the design cross sections.

\section{Study results}

Fig. 3 shows the result of calculating the volume fraction of water and air for the entire network as a whole for steady flow conditions. The constant pipeline filling up is observed in the network sections, except for the points of connecting the outlets and the supply header, where local level increase takes place due to pipeline flooding. Throughout the network there is a accompanying movement of the air along with the water flow due to the entraining ability (node 1 , node 2 ).

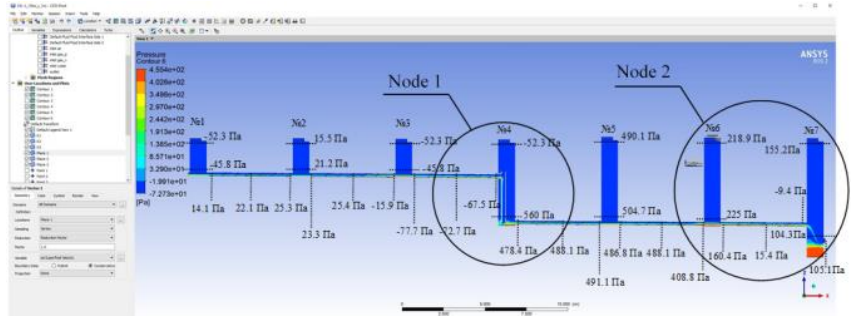

Fig. 3. Steady-state movement of two-phase water-air flow

Fig. 3 also shows the cross-sections (dashed lines) where the excess or vacuum pressure in the gas-air medium was recorded. In the direction of the flow to the pipe drop structure $($ No. 4,11$)$ the pressure in the under-roof space of pipelines and wells decreases due to increasing the entraining ability of the flow. After passing the drop structure on the main and supply header, the pressure in the under-roof space of the pipeline increases due to air injection. Fig. 4 shows that in main header sections 4-5 and 5-6, in inspection well No. 5, in the supply header section 11-6 there is an excess pressure. Further, after the flows confluence in well No. 6 , the entraining flow ability increases and the excess pressure decreases along the way to the SPS (No. 7). At the SPS inlet, a vacuum pressure of $-9.4 \mathrm{~Pa}$ is already present.

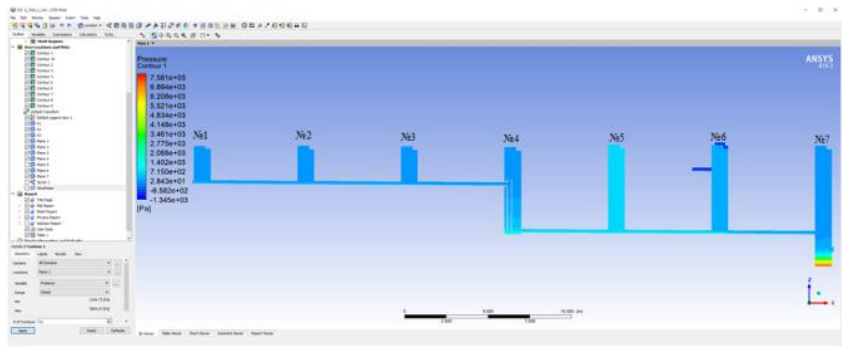

Fig. 4. Excess pressure field in the sewage network.

Fig. 5 shows the result of calculating the volume fraction of hydrogen sulfide in the air space of the sewage network. As you can see, gas accumulates most intensively in wells that receive runoff from outlets (Nos. 1, 3, 8, 9). Due to the flow drop from a height of $0.5 \mathrm{~m}$, the process of mass transfer with the air and further gas emission are intensified. Since the rates in the main network within these areas are low, the entraining ability does not allow pumping out all the generated gas into the network

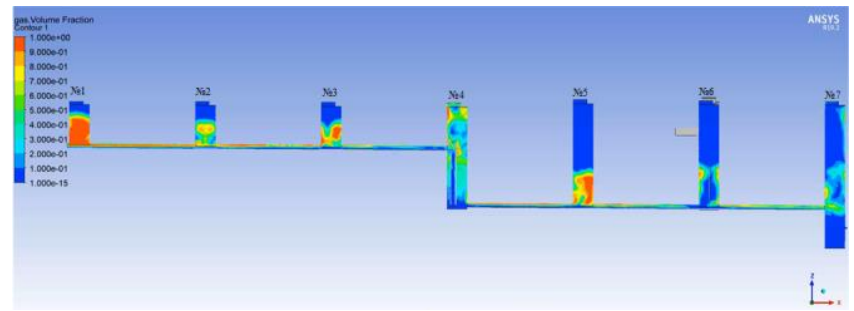

Fig. 5. The field of hydrogen sulfide volume fraction in the sewage network.

The conditions of wells natural ventilation through a house riser are not taken into account in this model. As well as the wells with pipe drop structure (Nos. 4, 11) can be referred to as the intensifiers of gas emission. In these wells there is a strong ejection by the flow of the gas-air mixture into the pipe drop structure. The flow fragmentation in the water well causes an intense release of hydrogen sulfide into the under-roof space of the well. In addition, large portions of gas are carried into the under-roof space of the discharge header and saturate the space of downstream wells (Nos. 5, 6). Due to the impinging jet formation the gas is also intensively generated in the SPS receiving tank.

\section{SUMMARY}

1. As a result of the work, the sewage network model has been developed that takes into account the combined movement of a wastewater stream and air-sewage gas (hydrogen sulfide) mixture entering the stream from the header tray.

2. The model calculation of the pressure field reflects the presence of vacuum pressure in the under-roof space of the header. A pressure value depends on the wastewater flow and header filling. The results qualitatively comply with the data of a full-scale experiment in the sewage network [15].

3. The model calculation of the field volume fraction allowed estimating the points of the sewage network with increased gas emission and accumulation of hydrogen sulfide.

4. A timely gas emission forecast depending on the hydraulic mode will allow the engineer to obtain the concentration, mass gas flow rate, as well as strong concentration accumulation locations. These data will make it possible to take timely measures to protect networks and structures most susceptible to gas corrosion, as well as to justify the decisions for gas purification.

\section{ACKNOWLEDGMENT}

The article was prepared as part of the work on the grant of the President of the Russian Federation NSh-4028.2018.6

\section{REFERENCES}

[1] Ilin, I., Levina, A., Iliashenko, O. Enterprise Architecture Analysis for Energy Efficiency of Saint-Petersburg Underground (2018) Advances in Intelligent Systems and Computing, 692, pp. 1214-1223.

[2] SP 32.13330.2012 Sewerage. Pipelines and Wastewater Treatment Plants. 
[3] L. Carrera, Caractérisation du transfert liquide/gaz du sulfure d'hydrogène dans les réseaux d'assainissement: These de doctorat de l'universite de Lyon, L. Carrera. Lyon: Ecole Doctorale de Chimie, 2016, $194 \mathrm{p}$.

[4] Y. C. Wang, A Dynamic Ventilation Model for Gravity Sewer Networks, Y. C. Wang, N. Nobi, T. Nguyen, L. Vorreiter, Water Science \& Technology, No. 65(1), 2011, pp. 60-66.

[5] S. Edwini-Bonsu, Air Flow in Sanitary Sewer Conduits due to Wastewater Drag: A Computational Fluid Dynamics Approach, S. Edwini-Bonsu, P. M. Steffler, Journal of Environmental Engineering Science, No. 3(5), 2004, pp. 331-342.

[6] S. Lowe, Sewer Ventilation Modeling, S. Lowe, Journal of water management modeling. 2017, https://www.chijournal.org/C415

[7] V.M. Vasiliev, Gas emission in drop structures and header sections when flowing wastewater inside, V.M. Vasiliev, O.M. Ilyina, New technologies and materials in underground construction Almanac of scientific and technical information, No. 1, 1995, pp. 3-7.

[8] A.V. Malkov, Calculation of the gaseous fluid amount flowing in deep-laid gravity sewage headers under the influence of the entraining liquid ability, A.V. Malkov, Civil Engineers Bulletin, No. 3(62), 2017, pp. 160-165.

[9] S. Lowe, Sewer Ventilation: Factors affecting airflow and modeling approaches, S. Lowe Journal of water management modeling, 2016, https://www.chijournal.org/C395
[10] S. Edwini-Bonsu, Modeling Ventilation Phenomenon in Sanitary Sewer Systems: A System Theoretic Approach, S. Edwini-Bonsu, P. M. Steffler, Journal of Hydraulic Engineering, No.132(8), 2006, pp. 778-790.

[11] S.V. Fyodorov, Assessment of hydrogen sulfide gas emission intensity at the section of the sewage network, S.V. Fyodorov, A.M. Telyatnikova, Materials of the International Research and Practical Conference dedicated to the 130th anniversary of the Water Use and Ecology Department "Current problems of water supply and disposal". SPbGASU, 2018, pp. 81-85.

[12] Makarov, V.M., Novikova, O.V., Tabakova, A.S. Energy efficiency in 'Green construction': Experience, issues, trends (2018) pp. 698-703

[13] A. Ilyinsky, M. Afanasyev, I. Ilin, M. Ilchenko, D. Metkin. An Economic Model of CO2 Geological Storage in Russian Energy Management System (2019) Advances in Intelligent Systems and Computing, 983, pp. 201-209.

[14] S.V. Fyodorov, Investigation of gas emission at the sewage network section, S.V. Fyodorov, V.M. Vasiliev, M. N. Klementiev, Water supply and sanitary engineering, No.5, 2019, pp. 54-60.

[15] A.A. Lukinykh and N.A. Lukinykh, Table for hydraulic calculation of sewage networks and drain siphons according to the formula of academician, N. N. Pavlovsky. 4th edition, enlarged. Moscow: Stroyizdat, 1974, p. 156 